\title{
Analysis of Rate One Quasi-Orthogonal Space-Time Block Coding over Rayleigh Fading Channel
}

\author{
Md. Abdullah Al Amin Ranju, Ragib Shakil Rafi \\ Electronics \& Communication Engineering, Khulna University, Khulna, Bangladesh \\ Email: ranju.ece@gmail.com, ku.08rafi@gmail.com
}

Received 26 April 2014; revised 26 May 2014; accepted 10 June 2014

Copyright (C) 2014 by authors and Scientific Research Publishing Inc.

This work is licensed under the Creative Commons Attribution International License (CC BY). http://creativecommons.org/licenses/by/4.0/

\section{(c) (i) Open Access}

\section{Abstract}

Full-rate is very important in any data transmission coding. For transmitting data at low bit rate than full-rate code, higher modulation scheme is required. But it is impossible to design full rate orthogonal designs with complex constellation for more than two transmit antennas. Only Alamouti code provides full-rate for two transmit antennas. In this paper, Bit Error Rate (BER) is calculated for Quasi-Orthogonal Space-time Block Coding (QOSTBC). Here we work with Rayleigh fading channel. We consider the codes which decodes pairs of symbols instead of simple separate decoding like Orthogonal Space-Time Block Coding. In Quasi-Orthogonal Space-time Block Code full-rate is achieved but full-diversity is sacrificed. Diversity is the most important techniques for providing reliable communication over fading channels. One of the diversity techniques that uses multiple transmit and/or receive antennas is space diversity. Multiple antenna technique provides a space diversity to struggle with the fading without necessarily sacrificing bandwidth resources, so the excellent solutions of removing the fading of the channel for broadband wireless communications is using space diversity. Then, with the constellation rotation of the symbol, rotated version of Quasi-Orthogonal Space-Time Block Code is generated. It provides full diversity. We simulate BER for QOSTBC, rotated QOSTBC, orthogonal STBC and for uncoded system. The simulation result shows that QOSTBC and rotated QOSTBC perform better than other systems. It shows that QOSTBC provides a full transmission rate but that rotated QOSTBC provides the full rate with the full diversity.

\section{Keywords}

Orthogonal Space-Time Block Coding, Quasi-Orthogonal Space-Time Block Coding, Rayleigh Fading, Rotated Quasi-Orthogonal Space-Time Block Coding 


\section{Introduction}

The transmission path in the wireless communication is air or free space for transmitting the electromagnetic signal to the destination. The transmitted signal may reach the receiver directly (Line-of-Sight) or through multiple reflections on local objects. When waves coming from these different paths are interacting with one another and for this received signal's amplitude and power fluctuates with time and if this fluctuation is rapid over a short period of time, then it is known as small-scale fading. The Rayleigh distribution [1] is commonly used to describe the statistical time varying nature of the received fading signal. The fading can be removed by using diversity and space diversity [2] is one of the diversity techniques that use multiple transmit and/or receive antennas. The possibility of deep fading over all paths' signals is greatly reduced by space diversity creating many independent propagation paths from transmitter to receiver. Information data are transmitted in a block-by-block fashion. Data transmission in an ST system is carried out in two dimensions, the space dimension and the time dimension, as the acronym ST suggests. The space dimension is spanned by multiple transmit-antennas while the time dimension is spanned by multiple time intervals over which multiple blocks are transmitted. By using STBC, QOSTBC [3] decreases the fading problem and provides partial diversity. But rotated QOSTBC [3] offers full-diversity. We simulate the BER and make a comparison for QOSTBC and rotated QOSTBC with OSTBC.

\section{Space-Time Block Codes}

We review the space-time block codes which provide maximum possible diversity for multiple transmit antennas in wireless communications. One example of space time block code is Alamouti code [3]. The generator matrix of this code to emphasize the indeterminate variables $x_{1}$ and $x_{2}$ in the design:

$$
\mathcal{G}\left(x_{1}, x_{2}\right)=\left(\begin{array}{cc}
x_{1} & x_{2} \\
-x_{2}^{*} & x_{1}^{*}
\end{array}\right)
$$

The Alamouti code is the only example of a full-rate full-diversity complex space-time block code using orthogonal design.

In general, a complex orthogonal space-time code is given by $T \times N$ generator matrix $\mathcal{G}$. Here $T$ and $N$ represents the number of time slots for transmitting one block of symbols and the number of transmit antennas respectively. The generator matrix $\mathcal{G}$ is chosen so that the rows and columns of each matrix are orthogonal to each other (i.e. the dot product of each row with another row is zero) and Orthogonal Design Theory provides this. Orthogonal Design Theory is two types, one for real numbers and other for complex numbers. Radon and Hurwitz [4] [5] provided the Real Orthogonal Designs and Complex Orthogonal Design is created from Real Orthogonal Design. The elements of the matrix are linear combinations of determinates $x_{1}, x_{2}, \cdots, x_{k}$ and their conjugates.

It is required that

$$
\mathcal{G}^{H} \mathcal{G}=p\left(\left|x_{1}\right|^{2}+\left|x_{2}\right|^{2}+\cdots+\left|x_{K}\right|^{2}\right) \boldsymbol{I}_{N}
$$

where, $\mathcal{G}^{H}$ is the Hermitian of $\mathcal{G}, \quad \boldsymbol{I}_{N}$ is the $N \times N$ identity matrix and pis a constant.The code rate of $\mathcal{G}$ is defined to be $R=K / T$. This is due to the fact that the code transmits $K$ constellation symbols in $T$ time slots. The rate is defined as the transmission rate relative to the maximum possible rate of a full-diversity code. It has been shown in [3] that the rate of a full-diversity code is less than or equal to one $R \leq 1$.

\section{Quasi-Orthogonal Space-Time Block Code}

\subsection{Encoding}

The encoding of QOSTBCs is very similar to the encoding of orthogonal STBCs. To transmit $b$ bits per time slot, we use constellations containing $2^{b}$ points. Using $4 b$ bits, constellation symbols $s_{1}, s_{2}, s_{3}, s_{4}$ are selected. Setting $x_{k}=s_{k}$ for $k=1,2,3,4$ in generator matrix $\mathcal{G}$, we arrive at a codeword matrix $\boldsymbol{C}=\mathcal{G}\left(s_{1}, s_{2}, s_{3}, s_{4}\right)$. Then, at time $t$, the four elements in the th row of $\boldsymbol{C}$ are transmitted from the four transmit antennas. Since four symbols $s_{1}, s_{2}, s_{3}, s_{4}$ are transmitted in four time slots presents a rate one code. 


\subsection{Transmission Model}

A wireless communication system is considered with $N$ transmit antennas and $M$ receive antennas [3]. The channel is assumed to be quasi-static slow flat fading and the path gain from transmit antenna $n$ to receive antenna $m$ is denoted by $\alpha_{n, m}$. The path gains are modeled as samples of independent complex Gaussian random variables. The variance is 0.5 for both imaginary part and real part of path gain. The channel is quasi-static so that the path gains are constant over a time frame of length $T$ and vary independently from one frame to another. Channel estimation is done with training/pilot sequences in regular intervals during the transmission. The received signal $r_{t, m}$ at time $t$ and antenna $m$ is given by,

$$
r_{t, m}=\sum_{n=1}^{N} \alpha_{n, m} C_{t, n}+\eta_{t, m}
$$

where, the noise samples $\eta_{t, m}$ are independent samples of a zero-mean complex Gaussian random variable and the code symbol $C_{t, n}$ is transmitted from $n$ transmit antenna at time $t$. The real part and imaginary part of noise symbols have equal variance $N /(2 \mathrm{SNR})$. The average energy of the transmitted symbols from each antenna is normalized to be 1 , so that the average power of the received signal at each receive antenna is $N$ and the signal-to-noise ratio is SNR.

\subsection{Decoding Algorithm}

Assuming perfect channel state information is available. So the receiver computes the decision metric [6],

$$
D=\sum_{t=1}^{T} \sum_{j=1}^{M}\left|r_{t, j}-\sum_{i=1}^{N} \alpha_{i, j} C_{t, i}\right|^{2}
$$

Overall possible $x_{k}=s_{k} \in C$ and decides in favor of the constellation symbols $s_{1}, s_{2}, \cdots, s_{k}$ that minimized the sum. We get from Alamouti code [3],

$$
\mathcal{G}=\left(\begin{array}{cc}
x_{1} & x_{2} \\
-x_{2}^{*} & x_{1}^{*}
\end{array}\right)
$$

Now let us consider $N=T=K=4$. So the generator matrix,

$$
\begin{aligned}
\mathcal{G}_{444} & =\left(\begin{array}{ccc}
\mathcal{G}\left(x_{1}, x_{2}\right) & \mathcal{G}\left(x_{3}, x_{4}\right) \\
-\mathcal{G}^{*}\left(x_{3}, x_{4}\right) & \mathcal{G}^{*}\left(x_{1}, x_{2}\right)
\end{array}\right) \\
& =\left(\begin{array}{cccc}
x_{1} & x_{2} & x_{3} & x_{4} \\
-x_{2}^{*} & x_{1}^{*} & -x_{4}^{*} & x_{3}^{*} \\
-x_{3}^{*} & -x_{4}^{*} & x_{1}^{*} & x_{2}^{*} \\
x_{4} & -x_{3} & -x_{2} & x_{1}
\end{array}\right)
\end{aligned}
$$

It is easy to see that the minimum rank of matrix $\mathcal{G}_{444}\left(s_{1}-\tilde{s}_{1}, s_{2}-\tilde{s}_{2}, s_{3}-\tilde{s}_{3}, s_{4}-\tilde{s}_{4}\right)$ is two [2]. The matrix constructed from $\mathcal{G}$ by replacing $x_{i}$ with $s_{i}-\tilde{s}_{1}$ is 2 . Therefore diversity of $2 \mathrm{M}$ is achieved and code rate $=1$. Now, if we define $v_{i}, i=1,2,3,4$ as the ith column of $\mathcal{G}$, it is easy to see that,

$$
\left\langle v_{1}, v_{2}\right\rangle=\left\langle v_{1}, v_{3}\right\rangle=\left\langle v_{2}, v_{4}\right\rangle=\left\langle v_{3}, v_{4}\right\rangle=0
$$

where, $\left\langle v_{i}, v_{j}\right\rangle=\sum_{l=1}^{4}\left(v_{i}\right)_{l}\left(v_{j}\right)_{l}^{*}$ is the inner product of vector $v_{i}$ and $v_{j}$. The subspace created by $v_{1}$ and $v_{4}$ is orthogonal to the subspace created by $v_{2}$ and $v_{3}$. Using this orthogonality, the maximum-likelihood decision metric (4) is equivalent to minimizing these two terms independently. The ML decision metric can be calculated as the sum of two terms $f_{14}\left(x_{1}, x_{4}\right)+f_{23}\left(x_{2}, x_{3}\right)$, where, $f_{14}$ is independent of $x_{4}$ and $x_{3}$ and $f_{23}$ is independent of $x_{1}$ and $x_{4}$. Thus the minimization of these two terms independently. Other words, first the decoder finds the pair $\left(s_{1}, s_{4}\right)$ that minimizes $f_{14}\left(x_{1}, x_{4}\right)$ among all possible values of $\left(x_{1}, x_{4}\right)$ pair. Then, or in parallel, the decoder selects pair $\left(s_{2}, s_{3}\right)$ similarly. Simple manipulation of Equation (4) becomes, 


$$
\begin{aligned}
f_{14}\left(x_{1}, x_{4}\right)= & \sum_{m=1}^{M}\left(\left(\sum_{n=1}^{4}\left|\alpha_{n, m}\right|^{2}\right)\left(\left|x_{1}\right|^{2}+\left|x_{4}\right|^{2}\right)\right. \\
& +2 \Re\left\{\left(-\alpha_{1, m} r_{1, m}^{*}-\alpha_{2, m}^{*} r_{2, m}-\alpha_{3, m}^{*} r_{3, m}-\alpha_{4, m} r_{4, m}^{*}\right) x_{1}\right. \\
& +\left(-\alpha_{4, m} r_{1, m}^{*}+\alpha_{3, m}^{*} r_{2, m}+\alpha_{2, m}^{*} r_{3, m}-\alpha_{1, m} r_{4, m}^{*}\right) x_{4} \\
& \left.\left.+\left(\alpha_{1, m} \alpha_{4, m}^{*}-\alpha_{2, m}^{*} \alpha_{3, m}-\alpha_{2, m} \alpha_{3, m}^{*}+\alpha_{1, m}^{*} \alpha_{4, m}\right) x_{1} x_{4}^{*}\right\}\right) \\
f_{23}\left(x_{2}, x_{3}\right)= & \sum_{m=1}^{M}\left(( \sum _ { n = 1 } ^ { 4 } | \alpha _ { n , m } | ^ { 2 } ) ( | x _ { 2 } | ^ { 2 } + | x _ { 3 } | ^ { 2 } ) 2 \Re \left\{\left(-\alpha_{2, m} r_{1, m}^{*}-\alpha_{1, m}^{*} r_{2, m}-\alpha_{4, m}^{*} r_{3, m}-\alpha_{3, m} r_{4, m}^{*}\right) x_{2}\right.\right. \\
& +\left(-\alpha_{3, m} r_{1, m}^{*}+\alpha_{4, m}^{*} r_{2, m}+\alpha_{1, m}^{*} r_{3, m}-\alpha_{2, m} r_{4, m}^{*}\right) x_{3} \\
& \left.\left.+\left(\alpha_{2, m} \alpha_{3, m}^{*}-\alpha_{1, m}^{*} \alpha_{4, m}-\alpha_{1, m} \alpha_{4, m}^{*}+\alpha_{2, m}^{*} \alpha_{3, m}\right) x_{2} x_{3}^{*}\right\}\right)
\end{aligned}
$$

where, the real part of a is denoted by $\mathfrak{R}\{a\}$. It is clear that decoding pairs of symbols for the QOSTBCs is more complex than decoding single symbols for the space time block codes.

\section{Rotated Quasi-Orthogonal Space-Time Block Code}

The minimum rank of the difference matrix $\boldsymbol{D}\left(C^{i}, C^{j}\right)$ is two for QOSTBCs in (7) for regular symmetric constellations same as PSK and QAM. For M receive antennas, a diversity of $2 \mathrm{M}$ is achieved while the code rate is one. The minimum diversity of $4 \mathrm{M}$ for a rate one complex orthogonal code is impossible in this case if all symbols are chosen from the same constellation [3]. For different transmitted symbols if we use different constellations we get full diversity. This means that we may rotate half of the symbols before transmission. This is called Rotated Quasi-Orthogonal Space Time Block Codes (Rotated QOSTBCs). This code is very powerful because it provides the full diversity, rate one, and simple pair wise decoding with satisfactory performance. The encoding algorithm and the transmission model are same as the QOSTBCs.

\section{Decoding}

The generator matrix of QOSTBC from (7) is recall here,

$$
\mathcal{G}_{444}=\left(\begin{array}{cccc}
x_{1} & x_{2} & x_{3} & x_{4} \\
-x_{2}^{*} & x_{1}^{*} & -x_{4}^{*} & x_{3}^{*} \\
-x_{3}^{*} & -x_{4}^{*} & x_{1}^{*} & x_{2}^{*} \\
x_{4} & -x_{3} & -x_{2} & x_{1}
\end{array}\right)
$$

For rotated QOSTBC $x_{3}$ and $x_{4}$ are get from the rotated version of $x_{3}$ and $x_{4}$. They are rotated before transmission and is denoted by $\tilde{x}_{3}$ and $\tilde{x}_{4}$. Here, $\tilde{x}_{3}=\mathrm{e}^{j \varnothing} x_{3}$ and $\tilde{x}_{4}=\mathrm{e}^{j \varnothing} x_{4}$ and $\varnothing$ is the constellation rotation angle.

Now for rotated QOSTBC the generator matrix (10) becomes:

$$
\mathcal{G}_{444}=\left(\begin{array}{ccrc}
x_{1} & x_{2} & x_{3} \mathrm{e}^{j \varnothing} & x_{4} \mathrm{e}^{j \varnothing} \\
-x_{2}^{*} & x_{1}^{*} & -\left(x_{4} \mathrm{e}^{j \varnothing}\right)^{*} & \left(x_{3} \mathrm{e}^{j \varnothing}\right)^{*} \\
-\left(x_{3} \mathrm{e}^{j \varnothing}\right)^{*} & -\left(x_{4} \mathrm{e}^{j \varnothing}\right)^{*} & x_{1}^{*} & x_{2}^{*} \\
x_{4} \mathrm{e}^{j \varnothing} & -x_{3} \mathrm{e}^{j \varnothing} & -x_{2} & x_{1}
\end{array}\right)
$$

We can see that the decoding decision for symbols $x_{1}$ and $x_{4}$ are obtained by minimizing the metric $f_{14}$, similarly the decoding decision for symbol $x_{2}$ and $x_{3}$ are obtained by minimizing the metric $f_{23}$. Clearly decoding of $x_{1}$ and $x_{4}$ can be performed separately from the decoding of $x_{2}$ and $x_{3}$. Since $x_{1}$ and $x_{4}$ 
$\left(x_{2}\right.$ and $x_{3}$ ) are each a complex symbol their decoding requires the joint detection of two complex symbols (i.e. four real symbols) in total.

So, the simple manipulation of decision metric becomes:

$$
\begin{aligned}
f_{14}\left(x_{1}, x_{4}\right)= & \sum_{m=1}^{M}\left(\left(\sum_{n=1}^{4}\left|\alpha_{n, m}\right|^{2}\right)\left(\left|x_{1}\right|^{2}+\left|x_{4}\right|^{2}\right)\right. \\
& +2 \Re\left\{\left(-\alpha_{1, m} r_{1, m}^{*}-\alpha_{2, m}^{*} r_{2, m}-\alpha_{3, m}^{*} r_{3, m}-\alpha_{4, m} r_{4, m}^{*}\right) x_{1}\right. \\
& +\left(-\alpha_{4, m} r_{1, m}^{*}+\alpha_{3, m}^{*} r_{2, m}+\alpha_{2, m}^{*} r_{3, m}-\alpha_{1, m} r_{4, m}^{*}\right) x_{4} \mathrm{e}^{j \varnothing} \\
& \left.\left.+\left(\alpha_{1, m} \alpha_{4, m}^{*}-\alpha_{2, m}^{*} \alpha_{3, m}-\alpha_{2, m} \alpha_{3, m}^{*}+\alpha_{1, m}^{*} \alpha_{4, m}\right) x_{1}\left(x_{4} \mathrm{e}^{j \varnothing}\right)^{*}\right\}\right) \\
f_{23}\left(x_{2}, x_{3}\right)= & \sum_{m=1}^{M}\left(\left(\sum_{n=1}^{4}\left|\alpha_{n, m}\right|^{2}\right)\left(\left|x_{2}\right|^{2}+\left|x_{3}\right|^{2}\right)\right. \\
& +2 \Re\left\{\left(-\alpha_{2, m} r_{1, m}^{*}-\alpha_{1, m}^{*} r_{2, m}-\alpha_{4, m}^{*} r_{3, m}-\alpha_{3, m} r_{4, m}^{*}\right) x_{2}\right. \\
& +\left(-\alpha_{3, m} r_{1, m}^{*}+\alpha_{4, m}^{*} r_{2, m}+\alpha_{1, m}^{*} r_{3, m}-\alpha_{2, m} r_{4, m}^{*}\right) x_{3} \mathrm{e}^{j \varnothing} \\
& \left.\left.+\left(\alpha_{2, m} \alpha_{3, m}^{*}-\alpha_{1, m}^{*} \alpha_{4, m}-\alpha_{1, m} \alpha_{4, m}^{*}+\alpha_{2, m}^{*} \alpha_{3, m}\right) x_{2}\left(x_{3} \mathrm{e}^{j \varnothing}\right)^{*}\right\}\right)
\end{aligned}
$$

The decoding algorithm in (12) and (13) are more complex than the QOSTBC in (8) and (9). Where in (12) and (13) $x_{1}$ and $x_{2}$ are non-rotated complex constellation symbols. The rotated QOSTBC decoding algorithm in (13) and (13) can be decoded with joint detection of only two real symbols, while still achieving full transmit diversity and full code rate.

\section{Simulation Results}

In this section, we provide simulation results for the QOSTBC in (7) and the Rotated QOSTBC in (11) and compare it with the other codes. These codes have a definite code rate and use different modulation scheme depends on the transmission bit rate. So, we have used the suitable modulation scheme due to the transmission bit rate for different STBCs. In all simulation result we consider different types of transmit antenna and one receive antenna and a given transmission bit rate on the Rayleigh fading channel. Then using this result the performance of each QOSTBC is provided. For, a fair comparison the modulation type and code size are varied with a view to maintaining a constant bit rate. As a result proper combination of QOSTBC and constellations are very essential. There is a constellation diagram which contains $2^{b}$ point for uncoded system with one transmission antenna or a full-rate OSTBCs transmission bit rate is b bits/(sHz). For a QOSTBCs with rate $R$, the transmission bit rate is $\mathrm{Rb}$ bits/(sHz). Since only for real signal constellation a full-rate full-diversity code exists. For complex signal constellations full-rate OSTBCs exist only for two transmit antenna and this code is provided by Alamouti. This code has a great advantage that, one can transmit the desire transmission bit rate while only depends on the modulation techniques. If one wants to be transmit 1 bits/(sHz) he may use BPSK, for 2 bits/(s Hz) use QPSK and continues for higher modulation technique. But code rate less than one need higher modulation technique for same transmission bit rate that obtained by Alamouti code. For half rate code needs QPSK where Alamouti codes need BPSK.

In case of full-rate code one can transmit more bits than lower code rate by using same modulation scheme. This is why full-rate code is more efficient code than lower rate code. For complex constellation and more than two transmit antennas full-rate is possible only by QOSTBC. So it is clear that it is more efficient than regular OSTBC. But there is no advantage in using QOSTBC of (7) and BPSK that results in the transmission bit rate of $1 \mathrm{bits} /(\mathrm{sHz})$ because a full-rate full-diversity code exist for real signal constellation. So, here we consider the simulation result of transmission bit rate of $2 \mathrm{bit} /(\mathrm{sHz})$. Figure 1 shows the comparison among QOSTBC, rotated QOSTBC and OSTBC. Figure 2 shows the simulation results of varying transmit antennas using QOSTBCs.

Figure 1 shows the simulation result for OSTBC, QOSTBC and rotated QOSTBC of the transmission bit rate of 2 bit/(sHz). These graphs are plotted against Bit Error Probability versus Signal-to-Noise Ratio (SNR) in dB. 


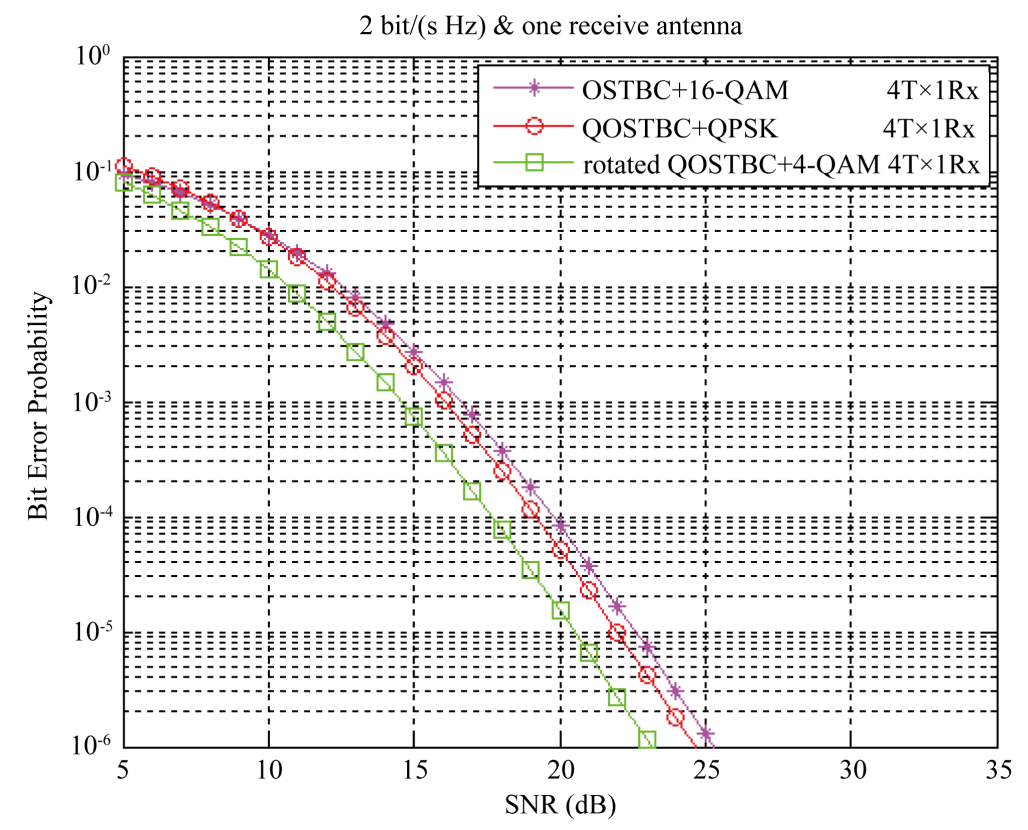

Figure 1. Bit error probability versus signal-to-noise ratio at 2 bits/(sHz).

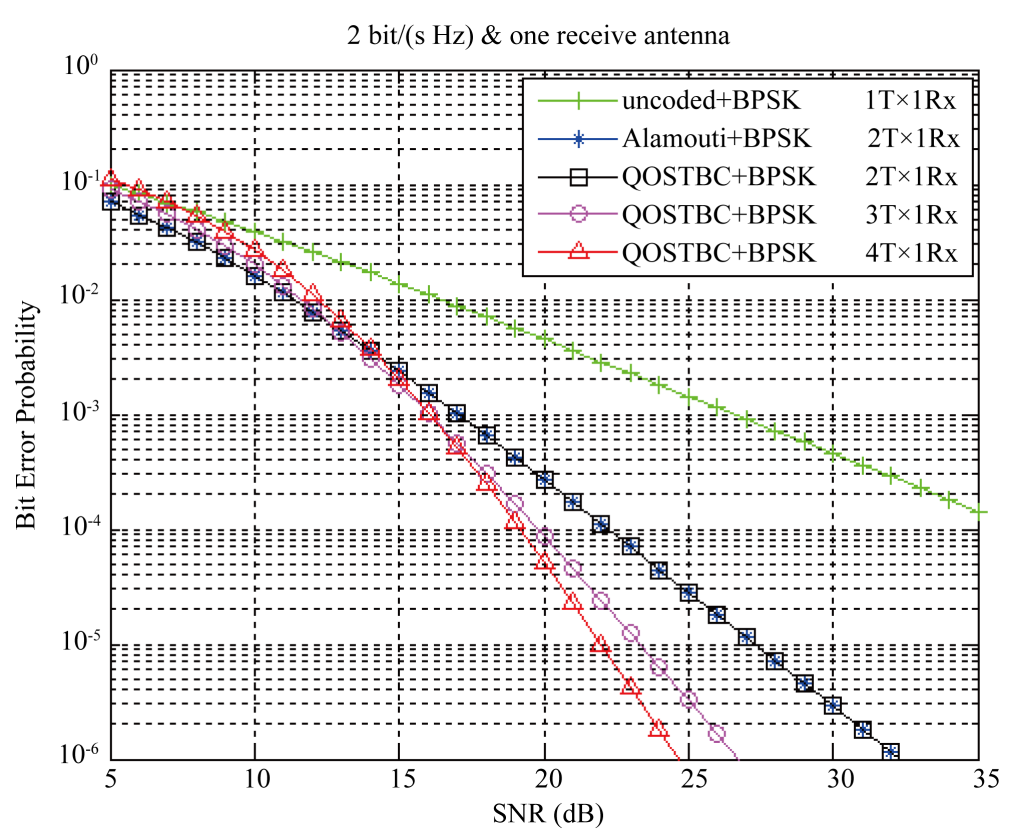

Figure 2. Bit error probability versus signal-to-noise ratio at $2 \mathrm{bits} /(\mathrm{sHz})$ with varying transmit antennas.

For transmission of the QOSTBC in (7) use QPSK modulation scheme, contains four message points. For OSTBC, it uses 16-QAM modulation scheme, contains sixteen message points and transmission bit rate is 2 bits/(sHz). Rotated QOSTBC uses 4-QAM modulation scheme, contains four message points.

At the reference point $10^{-4}$ QOSTBC provides about $0.7 \mathrm{~dB}$ less SNR than OSTBC. Rotated QOSTBC is about 1.4 dB less SNR than QOSTBC.

Therefore, full-rate QOSTBCs SNR is lower and rotated QOSTBCs SNR is the lowest. So, QOSTBC performs better than OSTBC and rotated QOSTBC performs best from OSTBC and QOSTBC for 2 bits/(sHz).

Figure 2 shows the comparison among the different number of transmit antennas for rate one code, any num- 
ber of antennas it uses QPSK modulation scheme for transmission bit rate $2 \mathrm{bit} /(\mathrm{sHz})$ and four message points.

For two transmit antenna, QOSTBC $\mathcal{G}_{444}$ code in (7) becomes $\mathcal{G}_{244}$ by removing of two columns.

$$
\therefore \mathcal{G}_{244}=\left(\begin{array}{cc}
x_{1} & x_{2} \\
-x_{2}^{*} & x_{1}^{*} \\
-x_{3}^{*} & -x_{4}^{*} \\
x_{4} & -x_{3}
\end{array}\right)
$$

The Alamouti code in (5) and the QOSTBC $\mathcal{G}_{244}$ code in (14) provide same curve in the graph so the performance of both codes are same for two transmit antennas.

The $\mathcal{G}_{444}$ code in (7) becomes a three transmit antenna QOSTBC $\mathcal{G}_{344}$ code by removing one column.

$$
\therefore \mathcal{G}_{344}=\left(\begin{array}{ccc}
x_{1} & x_{2} & x_{3} \\
-x_{2}^{*} & x_{1}^{*} & -x_{4}^{*} \\
-x_{3}^{*} & -x_{4}^{*} & x_{1}^{*} \\
x_{4} & -x_{3} & -x_{2}
\end{array}\right)
$$

The SNR of the four transmit antenna $\mathcal{G}_{444}$ code in (7) is about $0.7 \mathrm{~dB}$ less than $\mathcal{G}_{344}$ code in (15) and $\mathcal{G}_{344}$ code is about $2.25 \mathrm{~dB}$ less than the $\mathcal{G}_{244}$ code in (14). The uncoded curve is shown in the graph only for a fair comparison.

Therefore, if the number of transmit antenna increases, the SNR decreases. So, it performs better with increasing the number of transmit antenna.

\section{Conclusions}

Simulation results show that the QOSTBC performs better than OSTBC and rotated QOSTBC performs better than the QOSTBC. If the number of transmit antenna increases rate one code performs better. In fact, since the slope of the BER-SNR curve depends on the diversity, the QOSTBC curve also starts from a better point in the BER-SNR curve. It decodes the pair of symbol so decoding complexity is higher than the OSTBC but both codes have very low decoding complexity. The encoding complexity is very small and same. For two transmit antenna Alamouti scheme and QOSTBC provide same SNR and same BER.

So, the performance of QOSTBC and rotated QOSTBC is better than OSTBC. We work only with Rayleigh flat fading channel. So, there may also few options to works with fast or, slow fading channel or another fading channel like Rician or, Nakagami fading channel. Yet everybody works with four transmit antenna for QOSTBC. One can study for the maximum possible rate for a given number of transmit antenna that will provide full-rate and full-diversity.

\section{References}

[1] Simon, M.K. and Alouni, M.-S. (2000) Digital Communications over Fading Channels: A Unified Approach to Performance Analysis. John Wiley \& Sons, Ltd., Hoboken, 4-20, 70-265.

[2] Xiong, F.Q. (2000) Digital Modulation Techniques. Artech House, Inc., Boston.

[3] Jafarkhani, H. (2005) Space-Time Codes: Theory and Practice. Cambridge University Press, Cambridge, 1-125. http://dx.doi.org/10.1017/CBO9780511536779.004

[4] Proakis, J.G. (2001) Digital Communications. 4th Edition, McGraw-Hill, New York.

[5] Jafarkhani, H. (2001) A Quasi-Orthogonal Space-Time Block Code. IEEE Transactions on Communications, 49, 1-4. http://dx.doi.org/10.1109/26.898239

[6] Jankirman, M. (2004) Space-Time Codes and MIMO Systems. Artech House, Boston, London, 15-22, 47-50, 75-105. 